\title{
Star formation rate density and the stochastic background of gravitational waves
}

\author{
José C. N. de Araujo团 and Oswaldo D. Miranda ${ }^{1,2}$ 讨 \\ ${ }^{1}$ Divisão de Astrofísica - Instituto Nacional de Pesquisas Espaciais, \\ Avenida dos Astronautas 1758 - São José dos Campos - 12227-010 SP - Brazil and \\ ${ }^{2}$ Departamento de Física - Instituto Tecnológico de Aeronáutica, \\ Praça Marechal Eduardo Gomes 50 - São José dos Campos - 12228-900 SP - Brazil
}

(Dated: February 5, 2008)

\begin{abstract}
There is in the literature a number of papers addressing the stochastic background of gravitational waves (GWs) generated by an ensemble of astrophysical sources. The main ingredient in such studies is the so called star formation rate density (SFRD), which gives the density of stars formed per unit time. Some authors argue, however, that there is, in the equation that determines the amplitude of the stochastic background of GWs, an additional $(1+z)$ term dividing the SFRD, which would account for the effect of cosmic expansion onto the time variable. We argue here that the inclusion of this additional term is wrong. In order to clarify where the inclusion of the $(1+z)$ term is really necessary, we briefly discuss the calculation of event rates in the study of GRBs (gamma ray bursts) from cosmological origin.
\end{abstract}

PACS numbers: 04.30.Db, 98.70.Vc, 98.80.-k

\section{STOCHASTIC BACKGROUND OF GRAVITATIONAL WAVES}

Because of the fact the gravitational waves (GWs) are produced by a large variety of astrophysical sources and cosmological phenomena it is quite probable that the Universe is pervaded by a background of such waves.

We have recently shown [1] that the dimensionless amplitude of the stochastic background of GWs produced by an ensemble of sources is given by

$$
h_{\mathrm{BG}}^{2}=\frac{1}{\nu_{\mathrm{obs}}} \int h_{\text {single }}^{2} d R,
$$

where $h_{\text {single }}$ is the dimensionless amplitude produced by an event that generates a signal with observed frequency $\nu_{\mathrm{obs}}$; and $d R$ is the differential rate of production of GWs.

Eq.(1) is in fact a shortcut to the calculation of stochastic background of GWs. An interesting characteristic of this equation is that it is not necessary to know in detail the energy flux of the GWs produced at each frequency. If the characteristic values for the dimensionless amplitude and frequency are known and the event rate is given it is possible to calculate the stochastic background of GWs produced by an ensemble of sources.

In particular, for the case of a background produced by an ensemble of black holes, the differential rate reads

$$
d R_{\mathrm{BH}}=\dot{\rho}_{*}(z) \frac{d V}{d z} \phi(m) d m d z,
$$

where $\dot{\rho}_{*}(z)$ is the star formation rate density [hereafter SFRD (we refer the reader to the Appendix, where it is shown how the SFRD is obtained from observations);

\footnotetext{
*Electronic address: jcarlos@das.inpe.br
}

${ }^{\dagger}$ Electronic address: oswaldo@das.inpe.br in $\left.\mathrm{M}_{\odot} \mathrm{yr}^{-1} \mathrm{Mpc}^{-3}\right], d V$ is the comoving volume element and $\phi(m)$ is the initial mass function (IMF). In a few words, the $\phi(m) d m$ represents the number of stars per unit mass in the interval $[m, m+d m]$. The normalization of the IMF is obtained through the relation

$$
\int_{\mathrm{m}_{1}}^{\mathrm{m}_{\mathrm{u}}} m \phi(m) d m=1,
$$

where it is usually considered that $\mathrm{m}_{\mathrm{l}}=0.1 \mathrm{M}_{\odot}$ and $\mathrm{m}_{\mathrm{u}}=125 \mathrm{M}_{\odot}$ (see, e.g., Ref. [1]).

Some authors argue that there is an additional $(1+z)$ term in the equation for the differential rate dividing $\dot{\rho}_{\star}(z)$, which would take into account the effect of cosmic expansion onto the time variable.

In fact, there are two different points of view in the literature concerning the inclusion or not of the factor $(1+z)$. The first group does not include this factor dividing the SFRD (see, e.g., Refs. 1, 2, 3]). On the other hand, the second group argues that it is necessary to include the factor $(1+z)$ to account for the time dilation of the observed rate by cosmic expansion, converting a source-count equation to an event-rate equation (see, e.g., Refs. [4, 5, 6, 7]). We show here why such an additional term does not exist.

In order to address properly this issue, we should note firstly that the determination of the amplitude of the GWs is obtained from the flux received by a detector $\left(F_{\mathrm{GW}}\right)$. In this way, one divides the luminosity of the sources by $4 \pi d_{\mathrm{L}}^{2}$, where $d_{\mathrm{L}}$ is the so called luminosity distance. The luminosity obviously refers to the source frame. Since the luminosity depends on the differential rate, given, for example, by Eq.(2), the latter also refers to the source frame, therefore it is not necessary to redshift it. It is worth stressing that any necessary redshifting is taken into account in the definition of $d_{\mathrm{L}}$.

Another way to be convinced that the $(1+z)$ does not enter into the calculation of the differential rate of production of GWs, $d R_{\mathrm{BH}}$, is to derive Eq.(11) through a 
procedure completely different from that used in Ref.[1].

Let us write the specific flux received in GWs at the present epoch as (see, in particular, Eq. (15) in Ref. [8] and section 12.1 in Ref. $[$ ] $)$

$$
F_{\nu}\left(\nu_{\mathrm{obs}}\right)=\int \frac{l_{\nu}}{4 \pi d_{\mathrm{L}}^{2}} \frac{d \nu}{d \nu_{\mathrm{obs}}} d V
$$

where

$$
l_{\nu}=\frac{d L_{\nu}}{d V}
$$

is the comoving specific luminosity density (given, e.g, in $\operatorname{erg~s}^{-1} \mathrm{~Hz}^{-1} \mathrm{Mpc}^{-3}$ ), which obviously refers to the source frame.

As discussed in Refs. [8, [9], the above equations are valid to estimate a stochastic background radiation received on Earth independent of its origin. In the present paper $l_{\nu}$ can be written as follows

$$
l_{\nu}=\int \frac{d E_{\mathrm{GW}}}{d \nu} \dot{\rho}_{*}(z) \phi(m) d m,
$$

where $d E_{\mathrm{GW}} / d \nu$ is the specific energy of the source. Note that in the above equation $\dot{\rho}_{*}(z)$ refers to the source frame, therefore, there is not the putative $(1+z)$ factor responsible to the time dilation.

Thus, the flux $F_{\nu}\left(\nu_{\mathrm{obs}}\right)$ received on Earth reads

$$
F_{\nu}\left(\nu_{\mathrm{obs}}\right)=\int \frac{1}{4 \pi d_{\mathrm{L}}^{2}} \frac{d E_{\mathrm{GW}}}{d \nu} \frac{d \nu}{d \nu_{\mathrm{obs}}} \dot{\rho}_{*}(z) \phi(m) d m d V .
$$

Using Eq.(2) it follows that

$$
F_{\nu}\left(\nu_{\mathrm{obs}}\right)=\int \frac{1}{4 \pi d_{\mathrm{L}}^{2}} \frac{d E_{\mathrm{GW}}}{d \nu} \frac{d \nu}{d \nu_{\mathrm{obs}}} d R_{\mathrm{BH}} .
$$

Note that in the above equation, what multiplies $d R_{\mathrm{BH}}$ is nothing but the specific energy flux per unity frequency (in, e.g., $\mathrm{erg} \mathrm{cm}^{-2} \mathrm{~Hz}^{-1}$ ), i.e.,

$$
f_{\nu}\left(\nu_{\mathrm{obs}}\right)=\frac{1}{4 \pi d_{\mathrm{L}}^{2}} \frac{d E_{\mathrm{GW}}}{d \nu} \frac{d \nu}{d \nu_{\mathrm{obs}}}
$$

(see, e.g., Ref.[10]).

On the other hand, the specific energy flux per unit frequency for GWs is given by Ref. 11]

$$
f_{\nu}\left(\nu_{\mathrm{obs}}\right)=\frac{\pi c^{3}}{2 G} h_{\mathrm{BH}}^{2} .
$$

Also, the spectral energy density, the flux of GWs, received on Earth, $F_{\nu}$, in $\mathrm{erg} \mathrm{cm}^{-2} \mathrm{~s}^{-1} \mathrm{~Hz}^{-1}$ can be written from Refs. 12, 13] as

$$
F_{\nu}\left(\nu_{\mathrm{obs}}\right)=\frac{\pi c^{3}}{2 G} h_{\mathrm{BG}}^{2} \nu_{\mathrm{obs}}
$$

From the above equations one obtains

$$
h_{\mathrm{BG}}^{2}=\frac{1}{\nu_{\mathrm{obs}}} \int h_{\mathrm{BH}}^{2} d R_{\mathrm{BH}},
$$

which is nothing but the Eq. (1).

One could argue that what we derive here is nothing but a rehash of a well known equation for the calculation of electromagnetic backgrounds, found in textbooks on cosmology, applied to gravitational radiation backgrounds. But, since some authors in the GW community are erroneously introducing extra redshift factors in their calculations, it is worth presenting clearly how to calculate GW backgrounds of cosmological origin.

Also, these authors do not base their calculations either on the formulation of electromagnetic backgrounds presented in textbooks on cosmology or on an equation such as the one derived here. They inappropriately base their calculations on event rate equations, in which the time dilation needs to be considered. As a result, they consider that in other calculations involving the SFRD the redshifting should be taken into account.

It is worth stressing that the equation for the energy flux of GWs, we use in our derivation, is not obtained by adapting the equation for the Poynting flux by replacing the electric field with the GW amplitude, with the corresponding factors of $\mathrm{G}$ and c. This equation comes from general relativity.

One could argue when it is necessary to take into account the redshifting related to the time dilation. We now discuss a case where we must include the factor $(1+z)$ to take into account the time dilation of the observed rate.

The case is related to the Gamma Ray Bursts (hereafter GRBs), which are short and intense pulses of $\gamma$-rays, which last from a fraction of a second to several hundred seconds. It is worth mentioning that there are in the literature many papers dealing with the GRB event rates of cosmological origin, namely, Refs. [14, 15, 16, 17], among others.

The event rate for the GRBs of cosmological origin is related to the SFRD. The main reason to include the $(1+z)$ factor in this case is that we observe GRB events over a fixed time window $\Delta t_{\mathrm{obs}}$, which corresponds to $\Delta t_{\mathrm{obs}} /(1+z)$ in the source frame. Thus, the total number of GRBs can be written as (see, in particular, Ref. 14])

$$
N(>z)=\int_{z}^{\infty} \psi_{\mathrm{GRB}}\left(z^{\prime}\right) \frac{\Delta t_{\mathrm{obs}}}{\left(1+z^{\prime}\right)} \frac{d V}{d z^{\prime}} d z^{\prime},
$$

where $d V / d z$ is the comoving volume element and $\psi_{\mathrm{GRB}}(z)$ is the number of GRB events per comoving volume per unit time, which is proportional to $\dot{\rho}_{*}(z)$.

Generally speaking, in any event rate of cosmological sources, which involves a formation rate as a function of redshift or the like, the time dilation must be applied.

We see that the definition presented in Eq.(12) is completely different from the definition presented in Eq.(11). The misuse of the time dilation of the SFRD in the calculation of the energy flux is due to the fact that this involves a time rate, as the event rate of cosmological sources does. But, one has to bear in mind that the luminosity distance, that links the luminosity (source 
frame) and the energy flux (observer frame), is defined in such a way that any redshifting, including the time dilation, is implicity in its definition.

In the next section, we reinforce the argumentation against the inclusion of the extra $(1+z)$ term recalling how luminosity, flux and the luminosity distance are related to each other.

\section{LUMINOSITY, FLUX AND LUMINOSITY DISTANCE}

Although the material present in this section is standard, appearing in any textbook of cosmology, it is worth having a look at the relation between luminosity, flux and luminosity distance in order to recall that any redshifting, in particular the time dilation, is already taken into account.

An isotropic emission of photons (or gravitons in the present case) emitted by a source pass through spheres surrounding the source and, in the absence of expansion, the detected flux is exactly equal to the fraction of the area of the sphere surrounding the source and covered by the detector, that is, $d A / 4 \pi d_{\mathrm{L}}^{2}$ (where $d A$ is the area of the detector) times the luminosity $L$ of the source. In this case, $d_{\mathrm{L}}$ is simply the distance to the source (at this point we refer the reader to some textbook on cosmology as, e.g., [9, 18, 19]).

When the expansion of the Universe is taken into account, this consideration is slightly modified. In this case, we can use the Friedmann - Robertson - Walker (FRW) metric as being centered on the source; because of homogeneity, the comoving distance between the source and the observer is the same as we would calculate when we place the origin at the observer's location.

Setting $t=$ constant and $r=$ constant in the FRW line element, then we obtain

$$
d s^{2}=-r^{2} a^{2}\left(d \theta^{2}+\sin ^{2} \theta d \phi^{2}\right),
$$

where this equation represents the line element on the surface of a Euclidean sphere of radius $a r$, and $a=a(t)$ is the scale factor of the Universe.

The total energy emitted by a source per unit time, at the epoch $t_{1}$, over the bandwidth $(\nu, \nu+\Delta \nu)$ is given by

$$
d L=L J(\nu) d \nu
$$

where $J(\nu)$ is the intensity function.

In the case of a source emitting isotropically, when its light reaches us it is distributed uniformly across a sphere of coordinates $r=r_{1}$ centered on the source. However, we should note that for the source situated at comoving coordinate $r=r_{1}$ the photons (or gravitons) emitted at time $t_{1}$ will be detected at comoving coordinate $r=0$ at time $t_{0}$.

Note that because of the redshift, the arriving photons (or gravitons) with frequencies in the range $\left(\nu_{\mathrm{obs}}, \nu_{\mathrm{obs}}+\right.$
$\left.\Delta \nu_{\mathrm{obs}}\right)$ left the source in the frequency range $\left[\nu_{\mathrm{obs}}(1+\right.$ $\left.z),\left(\nu_{\mathrm{obs}}+\Delta \nu_{\mathrm{obs}}\right)(1+z)\right]$.

Thus, the quantity of energy that leaves the source (at $r=r_{1}$ ) between the times $t_{1}$ and $t_{1}+\Delta t_{1}$ in the above frequency range is simply obtained by

$$
L J\left(\nu_{\mathrm{obs}}[1+z]\right) \Delta \nu_{\mathrm{obs}}(1+z) \Delta t_{1} .
$$

In order to find the number of photons (or gravitons) that carry the above quantity of energy, we should divide Eq. (15) by the energy $(1+z) h \nu_{\mathrm{obs}}$, where $h$ is the Planck constant. That is

$$
\delta N=\frac{L J\left(\nu_{\mathrm{obs}}[1+z]\right) \Delta t_{1}}{h \nu_{\mathrm{obs}}} \Delta \nu_{\mathrm{obs}}
$$

At the time of reception, these photons (or gravitons) are distributed across a surface area $4 \pi r_{1}^{2} a^{2}\left(t_{0}\right)$ and they are received over a time interval $\left(t_{0}, t_{0}+\Delta t_{0}\right)$. Thus, the number of particles received by a detector per unit area held normal to the line of sight and per unit time is given by

$$
\frac{L J\left(\nu_{\mathrm{obs}}[1+z]\right)}{h \nu_{\mathrm{obs}}} \frac{\Delta t_{1}}{\Delta t_{0}} \frac{1}{4 \pi r_{1}^{2} a^{2}\left(t_{0}\right)} \Delta \nu_{\mathrm{obs}} .
$$

When received, each photon (or graviton) has been degraded in energy by the factor $1 /(1+z)$. Thus, the photons (gravitons) now has the energy $h \nu_{\mathrm{obs}}$. If we multiply Eq. (17) by this factor, we obtain the quantity of photons (gravitons) received per unit time by us across unit proper area held perpendicular to the line of sight to the source, and over a bandwidth $\left(\nu_{\mathrm{obs}}, \nu_{\mathrm{obs}}+\Delta \nu_{\mathrm{obs}}\right)$. Denoting this quantity $F_{\nu}\left(\nu_{\mathrm{obs}}\right) \Delta \nu_{\mathrm{obs}}$, we have

$$
F_{\nu}\left(\nu_{\mathrm{obs}}\right) \Delta \nu_{\mathrm{obs}}=L J\left(\nu_{\mathrm{obs}}[1+z]\right) \frac{\Delta t_{1}}{\Delta t_{0}} \frac{1}{4 \pi r_{1}^{2} a^{2}\left(t_{0}\right)} \Delta \nu_{\mathrm{obs}} .
$$

We should note that in the above equation $\Delta t_{1} / \Delta t_{0}=$ $1 /(1+z)$. This is just the $(1+z)$ factor claimed by some authors that should divide the SFRD to take into account the time dilation of the observed rate by cosmic expansion. As seen below this factor is already incorporated in the definition of the luminosity distance. Thus, finally we obtain

$$
F_{\nu}\left(\nu_{\mathrm{obs}}\right)=\frac{L J\left(\nu_{\mathrm{obs}}[1+z]\right)}{4 \pi r_{1}^{2} a^{2}\left(t_{0}\right)(1+z)},
$$

where $F_{\nu}\left(\nu_{\mathrm{obs}}\right)$ is the flux density.

In a few words, the above result can be understood as follows: The photons (gravitons) from the source pass through a sphere of proper area $4 \pi r_{1}^{2}$ on which the observer sits, where $r_{1}$ is the comoving distance. Moreover, redshift affects the flux density in four ways: photon (graviton) energy and arrival rates are redshifted, reducing the flux by a factor $(1+z)^{2}$; opposing this, the bandwidth $\Delta \nu$ is reduced by a factor $(1+z)$, so the energy flux per unit band goes down by one power of $(1+z)$; finally, the observed photons (gravitons) at 
frequency $\nu_{\mathrm{obs}}$ were emitted at frequency $\nu_{\mathrm{obs}}(1+z)$. Thus, the flux received on Earth from a cosmological source is the luminosity of the source divided by the total area and divided by $(1+z)$ as given in Eq. (19).

The bolometric flux can be obtained from the integration of Eq. (19) over all frequencies. The result is

$$
F_{\mathrm{bol}}=\frac{L_{\mathrm{bol}}}{4 \pi r_{1}^{2} a^{2}\left(t_{0}\right)(1+z)^{2}},
$$

where the quantity $d_{\mathrm{L}}=r_{1} a\left(t_{0}\right)(1+z)$ is the so called luminosity distance. It is worth stressing that Eq. (20) is a direct consequence of the conservation of energy.

\section{CONCLUDING REMARKS}

The $(1+z)$ dividing the SFRD, which some authors claim would account for the time dilation of the observed rate by cosmic expansion, converting a source-count equation to an event-rate equation (see, e,g, 4, 5, 6, 7]), is nothing but the arrival rates, which is implicit in the luminosity distance. In particular, Refs. 2, 3] implicitly corroborate with the argumentation given here.

It is important to stress that for the cosmological background of GW models we studied in Ref. [20], the inclusion of the $(1+z)$ term would reduce by a factor of $\sim 3-5$ the signal-to-noise ratio (SNR) predicted for the LIGO observatories. For some models, this would mean to predict a non detection of such a stochastic background of GWs.

We hope this brief report contributes to clarify the reader why in the calculation of the amplitude of a stochastic background of GWs, from cosmological sources, it is not necessary to redshift the SFRD as some authors are arguing for; and also why the GRB community, or any one who calculates cosmological event rates, should keep using the time dilation in their calculations.

\section{Acknowledgments}

J.C.N.A. would like to thank the Brazilian agency $\mathrm{CNPq}$ for partial support (grant 303868/2004-0). O.D.M. would like to thank the Brazilian agency FAPESP for support (grants 02/07310-0 and 02/015284). We would like also to thank the referees for useful comments and suggestions.

\section{APPENDIX: THE STAR FORMATION RATE DENSITY}

The SFRD is inferred from observations of the light emitted by stars at various wavelengths. In particular, 21] investigated the galaxy luminosity density of restframe ultraviolet (UV) radiation up to $z \sim 4$, and they converted it into the SFRD. The rest-frame UV light is considered to be a direct tracer of star formation because it is mainly radiated by short-lived massive stars.

These observable samples are flux-limited, and thus the intrinsic luminosity of the faintest objects in the sample changes with redshift. This incompleteness of the samples is corrected by using a functional (Schechter function) to the luminosity function obtained from the observations themselves. Then, the conversion from luminosity density to SFRD generally relies on stellar population models, an assumed star formation history and a specific choice for the IMF (generally, it is considered the Salpeter IMF).

In this way, the SFRD is mainly derived from the observed luminosity at high redshift, that is, $\dot{\rho}_{*}(z) \propto L$.
[1] J.C.N. de Araujo, O.D. Miranda and O.D. Aguiar, Phys. Rev. D 61, 124015 (2000).

[2] V. Ferrari, S. Matarrese and R. Schneider, Mon. Not. R. Astron. Soc. 303, 247 (1999).

[3] T. Regimbau and J.A. de Freitas Pacheco, Astron. Astrophys. 376, 381 (2001).

[4] R. Schneider, A. Ferrara, B. Ciardi, V. Ferrari and S. Matarrese, Mon. Not. R. Astron. Soc. 317, 385 (2000).

[5] D. M. Coward, R.R. Burman and D.G. Blair, Mon. Not. R. Astron. Soc. 324, 1015 (2001).

[6] D. M. Coward, M.H.P.M. van Putten and R.R. Burman, Astrophys. J. 580, 1024 (2002).

[7] D. M. Coward, R. R. Burman and D. G. Blair Mon. Not. R. Astron. Soc. 329, 411 (2002).

[8] A. J. Farmer and E. S. Phinney, Mon. Not. R. Astron. Soc. 346, 1197 (2003).

[9] J. A. Peacock, in Cosmological Physics (Cambridge University Press, Cambridge, England, 1999).

[10] V. Ferrari, R. Schneider and S. Matarrese, Mon. Not. R.
Astron. Soc. 303, 258 (1999).

[11] B. J. Carr, Astron. Astrophys. 89, 6 (1980).

[12] D. H. Douglass and V. G. Braginsky, in General Relativity: An Einstein Centenary Survey, edited by S. W. Hawking and W. Israel (Cambridge University Press Cambridge, England, 1979), p. 90.

[13] D. Hils, P. L. Bender, and R. F. Webbink, Astrophys. J. 360, 75 (1990).

[14] V. Bromm and A. Loeb, Astrophys. J. 575, 111 (2002).

[15] T. Totani and A. Panaitescu, Astrophys. J. 576, 120 (2002).

[16] C. Firmani, V. Avila-Reese, G. Ghisellini and A. V. Tutukov, Astrophys. J. 611, 1033 (2004).

[17] C. Porciani and P. Madau, Astrophys. J. 548, 522 (2001).

[18] E. W. Kolb and M. S. Turner, in The Early Universe (Addison Wesley Pub. Comp., USA, 1994).

[19] J. V. Narlikar, in Introduction to Cosmology (Cambridge University Press, Cambridge, England, 1993).

[20] J. C. N. de Araujo, O. D. Miranda and O. D. Aguiar, 
Class. Quantum Grav. 21, S545 (2004).

[21] P. Madau, H. C. Ferguson, M. E. Dickinson, M. Giavalisco, C. C. Steidel and A. Fruchter, Mon. Not. R.
Astron. Soc. 283, 1388 (1996). 